\title{
Comparative analysis of response modes for gold nanoparticle biosensor based on localized surface plasmon resonance
}

\author{
A. Lopatynskyi ${ }^{1}$, O. Lopatynska ${ }^{2}$, V. Chegel ${ }^{1}$ \\ ${ }^{1}$ V. Lashkaryov Institute of Semiconductor Physics, National Academy of Sciences of Ukraine, \\ 41, prospect Nauky, 03028 Kyiv, Ukraine, Phone: +38 (044) 525-56-26 \\ E-mail:lop2000@ukr.net,vche111@yahoo.com \\ ${ }^{2}$ Taras Shevchenko National University of Kyiv, build. 1, 2, Academician Glushkov prospect, \\ 03022 Kyiv, Ukraine, Phone: +38 (044) 526-22-96, e-mail: olga_lopatynska@ukr.net
}

\begin{abstract}
The theoretical comparison of possible response measurement techniques for a biosensor based on localized surface plasmon resonance (LSPR) in spherical Au nanoparticle was made. The methods for measuring LSPR response considered differed in the treatment of the change of light extinction spectrum along the wavelength and extinction coordinate axes upon the formation of dense biomolecular monolayer on the surface of the sensitive element. In addition, the transformation of the extinction LSPR band with increase of the nanoparticle radius from 5 to $125 \mathrm{~nm}$ was investigated towards the optimization of LSPR response for dipolar and quadrupolar LSPR extinction peaks. A novel method for the measurement of wavelength shift $H_{\text {top }}^{+}$was introduced and demonstrated to be more effective for estimation of the LSPR biosensor response as compared to commonly measured extinction peak shift $\Delta \lambda_{\max }$. These techniques were proved to produce maximal LSPR response when large-size $\mathrm{Au}$ nanoparticle (with a radius of $125 \mathrm{~nm}$ ) was used as a sensitive element of biosensor. The preferable mode of extinction difference measurement turned out to be $V_{\text {right }}$, which is carried out at a wavelength of the extreme extinction spectrum derivative on a right slope of the LSPR peak. For this method, optimal nanoparticle radii were found to be about $40 \mathrm{~nm}$ for the dipolar LSPR peak and near 100-105 nm for the quadrupolar one.
\end{abstract}

Keywords: localized surface plasmon resonance, gold nanoparticle, biosensor, response.

Manuscript received 27.04.10; accepted for publication 02.12.10; published online 28.02.11.

\section{Introduction}

Development of biosensors that make use of the localized surface plasmon resonance (LSPR) phenomenon in nanoscale structures of noble metals for transducing the biomolecular processes near the metal surface into the measurable optical response, and search for the new applications of such biosensors have been carried out widely during last years [1-8]. This considerable attention is explained by weighty advantages provided by this technique, among which are real-time label-free biomolecular detection $[9,10]$ and temperate measuring equipment requirements - response can be registered using simple UV-Vis spectrophotometer.
The nature of LSPR originates from the collective properties of conduction electrons contained in nanosized noble metal particle. High concentration of free electron gas enables resonant excitation of its collective oscillation with incident UV, visible or NIR light depending on the particle's material $[11,12]$ and the geometrical parameters of the nanostructure $[11,13-$ 15]. Such electronic response gives rise to unusual optical properties of nanoscale metal - a peak arises in the light extinction spectrum of the noble metal nanoparticles that is absent in the corresponding spectrum for the bulk material. The shape and position of the nanostructure's LSPR extinction peak are dependent on the dielectric properties of the surrounding medium, which also includes any possible overlayers [16-19]. This important characteristic feature promotes 
the development of an ambient medium refractive index sensor or a biosensor that can detect the emergent biomolecular coatings on the nanoparticle surface.

A problem in selection of LSPR response acquisition mode that will provide optimal performance arises during the process of the LSPR biosensor elaboration. The main issue is to choose the appropriate method for the treatment of the LSPR extinction peak in order to get the maximal response value for the same investigated process. Usually, two LSPR response techniques are applied: the measurement of the LSPR peak wavelength position shift [20-24] and the measurement of the extinction value on the selected wavelength within the peak [3, 9, 24-26]. Both methods possess inherent advantages and drawbacks that restrain their range of application. The first approach offers reliable detection of the biomolecular process because the characteristic shift of the light extinction peak position upon the formation of non-absorbing biomolecular coating on the metal nanoparticle can be induced only by the LSPR effect and it is weakly affected by the spectrophotometer calibration inaccuracy. On the other hand, if the biomolecular layer is rather thin, the shift of the peak position can be difficult to detect. The second technique provides more sensitive response to the biomolecular events in some situations and can be implemented easily for continuous monitoring [9], but it lacks the insensibility to calibration errors peculiar to the first one. Moreover, the search for an optimal wavelength position is needed for this method in order to maximize the measured response.

Recently, other techniques for the treatment of the LSPR extinction peak changes induced by the molecular adsorption on the surface of $\mathrm{Ag}$ and $\mathrm{Au}$ nanoparticles were reported that addressed the noise reduction of realtime LSPR signals. They include center-of-mass wavelength concept [24,27], calculation of total absolute differences between spectra [24], averaged absorbance differences [28] and LSPR band integration [25]. Application of these methods during the measurement of LSPR response enhanced the detection capabilities of LSPR sensors due to significant decrease of the LSPR kinetic curve noise [24, 25].

In this study, we conducted a comparative analysis of the possible modes of LSPR response measurement upon the formation of saturated biomolecular overlayers on the surface of the spherical gold nanoparticle used as a sensing element of LSPR biosensor. The dependences of the LSPR response on nanoparticle size and biomolecular shell thickness were theoretically calculated. Optimal nanoparticle size ranges for specific measurement modes were determined, and the most promising response measurement directions were highlighted. The LSPR sensing performance of dipole and quadrupole extinction peaks that are exhibited by large $\mathrm{Au}$ nanoparticles were also brought into comparison for the nanoparticle radius range from 80 to $125 \mathrm{~nm}$.

\section{Theory}

The LSPR biosensor system studied was composed of a spherical Au nanoparticle overlaid by a dense monolayer of globular biomolecules (approximated by solid balls), which was located in water (Fig. 1).

Optical constants of bulk Au in the range from 450 to $1300 \mathrm{~nm}$ were taken from [29] and fitted using highorder polynomial with wavelength spacing of $1 \mathrm{~nm}$. Additionally, dielectric function of gold was modified depending on the nanoparticle size in the model of the electron mean free path reduction. The change was made by using the effective electron relaxation time as follows:

$\tau_{e f}(R)=\left(\tau_{\text {bulk }}^{-1}+A \frac{V_{f}}{R}\right)^{-1}$

where $\tau_{\text {bulk }}=9.3 \times 10^{-15} \mathrm{~s} \quad$ [30] is the electron relaxation time for massive gold, $V_{f}=1.4 \times 10^{6} \mathrm{~m} / \mathrm{s}$ [31] is the Fermi velocity for $\mathrm{Au}, R$ is the nanoparticle radius and $A$ is a constant taken equal to 1 [32-34]. This size-dependent electron relaxation time was then incorporated into dielectric function expression in the Drude-Lorentz model $[35,36]$ to substitute $\tau_{\text {bulk }}$ :

$\varepsilon_{1}(\omega, R)=\varepsilon_{1 b u l k}(\omega)+\frac{\omega_{p}^{2}}{\omega^{2}+\frac{1}{\tau_{b u l k}^{2}}}-\frac{\omega_{p}^{2}}{\omega^{2}+\frac{1}{\tau_{e f}^{2}(R)}}$,

$\varepsilon_{2}(\omega, R)=\varepsilon_{2 b u l k}(\omega)+\frac{\omega_{p}^{2}}{\omega}\left[\frac{\tau_{e f}(R)}{\omega^{2} \tau_{e f}^{2}(R)+1}-\frac{\tau_{\text {bulk }}}{\omega^{2} \tau_{b u l k}^{2}+1}\right]$,

where $\varepsilon_{1}$ and $\varepsilon_{2}$ are the real and imaginary parts of the dielectric function, $\omega$ is the light angular frequency, $\omega_{p}=1.37 \times 10^{16} \mathrm{rad} / \mathrm{s}$ [36] is the plasma frequency for bulk gold. Modified optical constants of Au nanoparticle were obtained using the following formulas [37]:

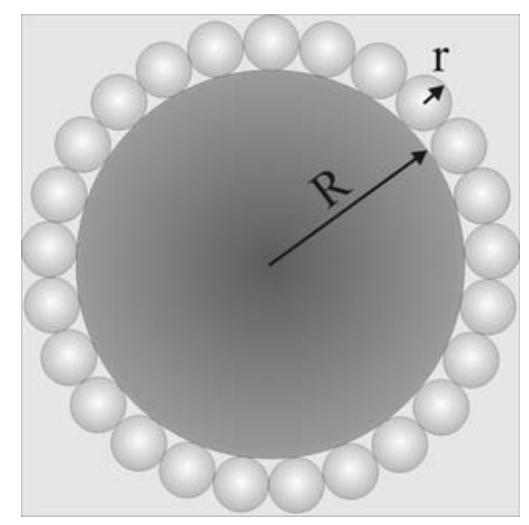

Fig. 1. Schematic drawing of the system under investigation. 


$$
\begin{aligned}
& n_{1}(\omega, R)=\sqrt{\frac{1}{2}\left[\varepsilon_{1}(\omega, R)+\sqrt{\varepsilon_{1}^{2}(\omega, R)+\varepsilon_{2}^{2}(\omega, R)}\right]}, \\
& k_{1}(\omega, R)=\sqrt{\frac{1}{2}\left[-\varepsilon_{1}(\omega, R)+\sqrt{\varepsilon_{1}^{2}(\omega, R)+\varepsilon_{2}^{2}(\omega, R)}\right]} .
\end{aligned}
$$

We have taken refractive index for biomolecules $n_{m}$ equal to $1.46[38,39]$. Refractive index for water was estimated by the equation adopted from [40]:

$$
n_{0}=1.32334+\frac{3479}{\lambda^{2}}-\frac{5.111 \times 10^{7}}{\lambda^{4}},[\lambda]=\mathrm{nm} \text {. }
$$

The calculation of light extinction efficiency of gold nanoparticle was carried out using Mie theory for a spherical particle with a shell [34] as follows:

$Q_{e x t}=\frac{2 \pi}{(|\vec{k}| R)^{2}} \sum_{l=1}^{L}(2 l+1) \operatorname{Re}\left(a_{l}+b_{l}\right)$,

where $\vec{k}$ is the light wave vector in the surrounding medium, $L$ is the number of multipole modes taken into account (estimated by the equation presented in [41]), $a_{l}$ and $b_{l}$ are Mie scattering coefficients for a coated sphere [34]. Parabolic approximation was used for accurate determination of the modelled spectra peak positions.

To consider a shell comprised of a dense monolayer of globular biomolecules, we applied symmetrical Bruggeman effective medium theory [34] that provides an effective value of the refractive index of the shell $n_{2}$ as a root of the equation

$$
f \frac{n_{m}^{2}-n_{2}^{2}}{n_{m}^{2}+2 n_{2}^{2}}+(1-f) \frac{n_{0}^{2}-n_{2}^{2}}{n_{0}^{2}+2 n_{2}^{2}}=0,
$$

where $f$ is a shell filling factor. It is clear that the filling factor for a saturated monolayer of spherical molecules on the surface of the nanoparticle is a function of a ratio between the nanoparticle diameter and shell thickness. The equation for the filling factor was derived from the following considerations. Let us imagine a spherical surface with radius of $R+r \quad(r$ is the biomolecule radius), where centres of the biomolecules are situated, and count the sections of biomolecules with this sphere. For this we approximate the location of these sections by the dense square lattice of circles with a radius of $r$ on a plane, thereby making it easy to obtain the formula for a number of biomolecules on the surface of the nanoparticle:

$$
N \approx \frac{S_{\text {plane }}}{S_{\text {square }}}=\frac{4 \pi(R+r)^{2}}{4 r^{2}}=\frac{\pi(R+r)^{2}}{r^{2}} .
$$

The filling factor can now be estimated as the ratio between the volume occupied by the biomolecules and the whole volume of the shell:

$$
f(R, r)=\frac{V_{m}}{V_{\text {shell }}}=\frac{N \cdot \frac{4}{3} \pi r^{3}}{\frac{4}{3} \pi\left[(R+2 r)^{3}-R^{3}\right]}=\frac{\pi r(R+r)^{2}}{(R+2 r)^{3}-R^{3}} .
$$

\section{Results and discussion}

The LSPR response measurement modes discussed in this paper are presented in Fig. 2. We introduced new methods of measuring the LSPR response that describe the difference between the LSPR spectra of a coated with biomolecules and bare nanoparticle in the following way. Firstly, we chose special points on the LSPR extinction spectrum of bare gold nanoparticle which would serve as a basis for the response measurement. We have selected the value of the derivative of the extinction efficiency $\frac{d Q}{d \lambda}$ as a criterion for the choice of these special points. According to this criterion, the points for the measurement of the LSPR response were fixed at wavelengths corresponding to $\frac{d Q}{d \lambda}=0$ and where $\frac{d Q}{d \lambda}$ has extrema in the LSPR peak wavelength range that corresponded to the peak maximum and two points on the left and right slopes of the LSPR extinction peak. Secondly, we laid down the LSPR responses for each of the special points as a distance between a point on the extinction spectrum corresponding to a bare $\mathrm{Au}$ nanoparticle and a point on the same spectrum corresponding to a coated nanoparticle in the horizontal (i.e. along the wavelength coordinate axis, designated as $H_{\text {left }}, H_{\text {top }}^{-}, H_{\text {top }}^{+}$and $H_{\text {right }}$ ) and vertical (i.e. along the extinction efficiency coordinate axis, designated as $V_{\text {left }}, \quad V_{\text {top }}$ and $V_{\text {right }}$ ) directions (see Fig. 2). Additionally, we calculated commonly used extinction peak shift $\Delta \lambda_{\text {max }}$ and maximal extinction shift $V_{\text {max }}$ in order to compare these to the previously described LSPR responses.

Additionally, we investigated the LSPR extinction spectrum transformation during the increase of the $\mathrm{Au}$ nanoparticle size. It was found out that when the nanoparticle radius reached $80 \mathrm{~nm}$, a new extinction peak corresponding to quadrupolar LSPR excitation emerged, which grew up with increasing nanoparticle size and overtopped the dipolar LSPR peak when nanoparticle radius came up to $125 \mathrm{~nm}$ (see Fig. 3). Thus, we also decided to check if the quadrupolar peak would offer higher LSPR response for large $\mathrm{Au}$ nanoparticles used as sensitive elements of LSPR biosensor in comparison with the dipolar one.

During the analysis of LSPR response modes described above for $\mathrm{Au}$ nanoparticle with a radius ranging from 5 to $125 \mathrm{~nm}$ covered by a saturated biomolecular overlayer with a biomolecule size of 2,5 , 
10, 15 and $20 \mathrm{~nm}$, the following peculiarities were discovered. Concerning the "horizontal" response modes $H_{\text {left }}, H_{\text {top }}^{-}, H_{\text {top }}^{+}, H_{\text {right }}$ and $\Delta \lambda_{\text {max }}$ for both dipolar and quadrupolar LSPR extinction peaks, we revealed that the quadrupolar peak yielded lower response values for the same core-shell configuration compared to the dipolar one (e.g. Fig. 4 compares $\Delta \lambda_{\text {max }}$ response values for dipolar and quadrupolar peaks). With respect to the dipolar LSPR peak, the maximal response values for all biomolecule sizes under study turned out to be $H_{\text {top }}^{-}$and $H_{\text {top }}^{+}$(Fig. 5), taking into account their opposite sign, but the maximal absolute value of the response was provided by $H_{t o p}^{+}$response measurement mode. This result suggests that an alternative method of "horizontal" LSPR response estimation $\left(H_{\text {top }}^{+}\right)$exists that can yield significantly larger response value than generally used $\Delta \lambda_{\max }$ measurement mode, which enables the improvement of the detection capability of the LSPR biosensor. For example, Au nanoparticle with a radius of $125 \mathrm{~nm}$ produces $\Delta \lambda_{\text {max }}=2.1 \mathrm{~nm}$ and $H_{\text {top }}^{+}=35 \mathrm{~nm}$ when coated by $2-\mathrm{nm}$ biomolecular layer that makes up about 17 times response enhancement. It should also be noted that the maximal LSPR response within the studied nanoparticle size range for all considered overlayer thicknesses in the $\mathrm{H}_{\text {top }}^{-}, \mathrm{H}_{\text {top }}^{+}$and $\Delta \lambda_{\text {max }}$ measurement modes is provided by $\mathrm{Au}$ nanoparticle with a radius of $125 \mathrm{~nm}$ that emphasizes the advantage of large-size nanoparticles as a basis of LSPR biosensor.

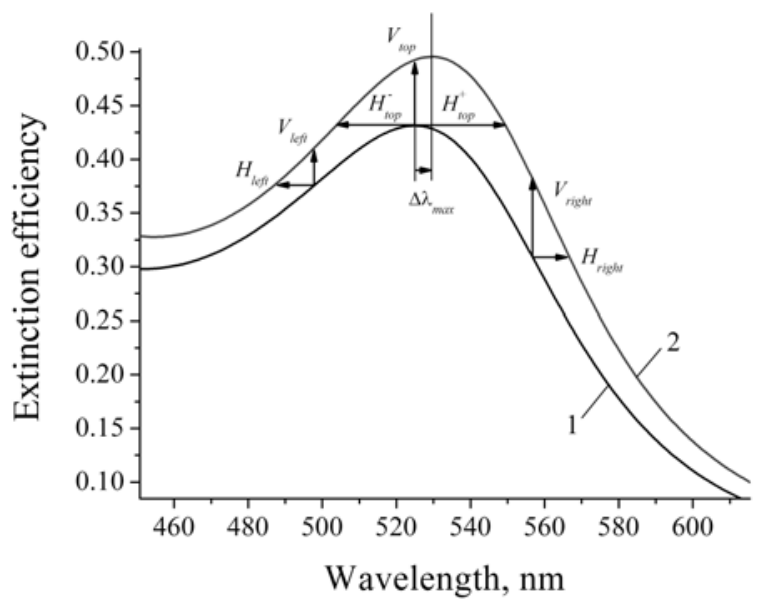

Fig. 2. LSPR response measurement modes for a biosensor response on biomolecular coating emergent on the $\mathrm{Au}$ nanoparticle surface: 1 - extinction efficiency spectrum for bare $\mathrm{Au}$ nanoparticle with a radius of $5 \mathrm{~nm}$ located in water, 2 - extinction efficiency spectrum for Au nanoparticle with a radius of $5 \mathrm{~nm}$ with $20 \mathrm{~nm}$ thick biomolecular coating located in water.

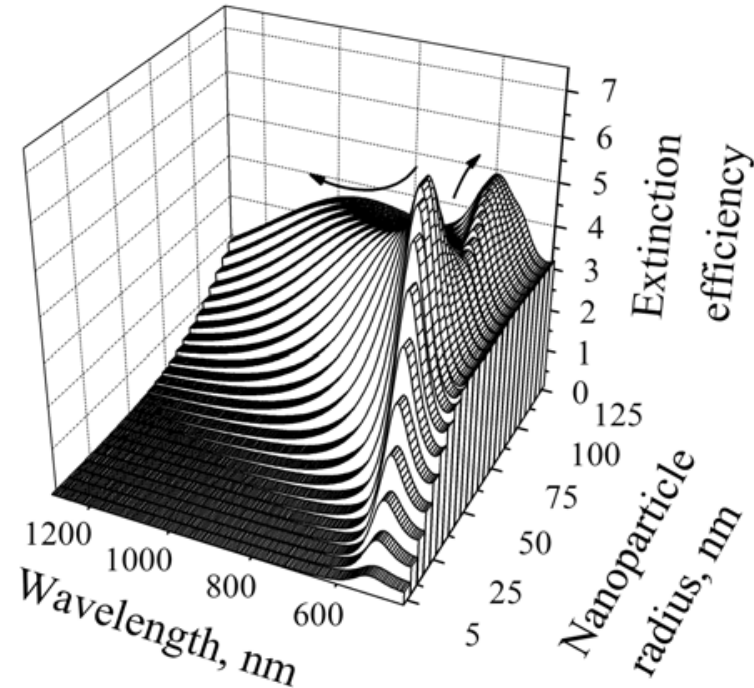

Fig. 3. Evolution of the extinction efficiency spectrum upon the increase of size of bare $\mathrm{Au}$ nanoparticle located in water. Arrows show the course of extinction peaks transformation.
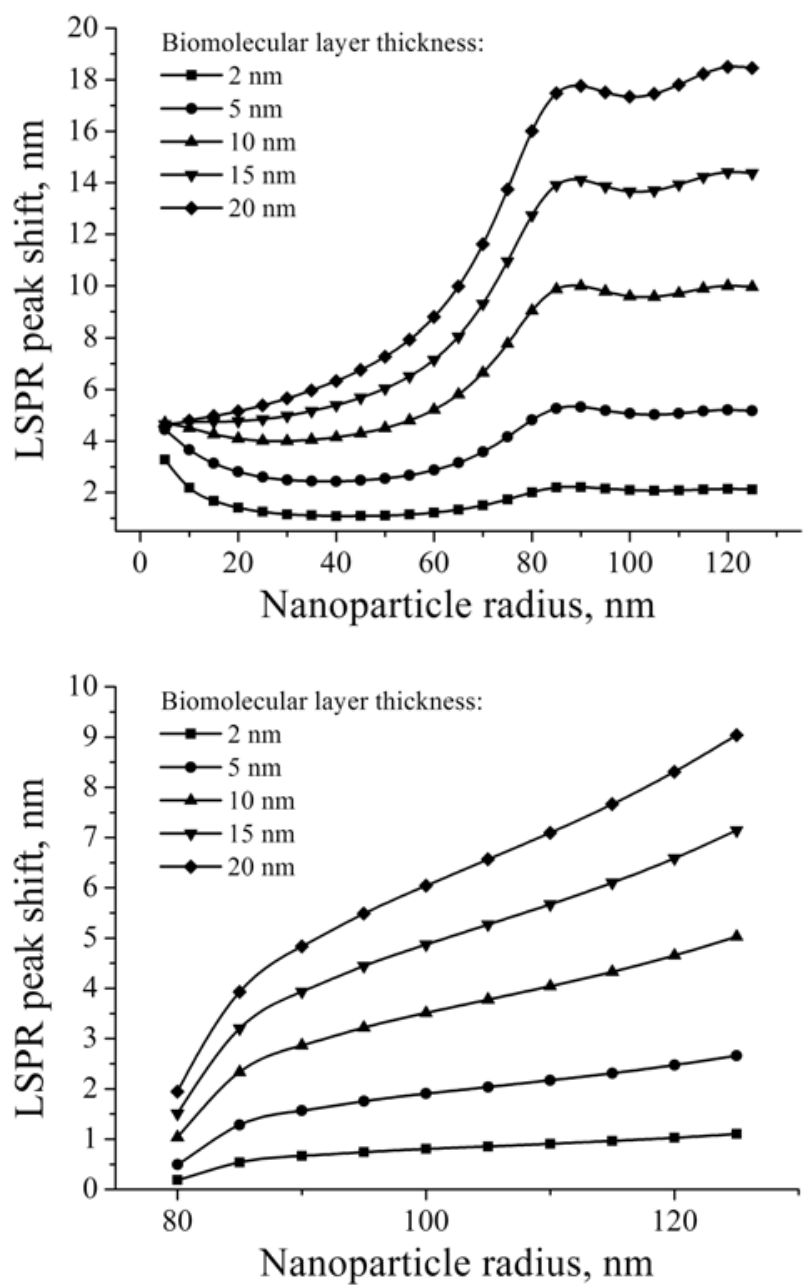

Fig. 4. LSPR extinction peak shift $\Delta \lambda_{\max }$ versus nanoparticle radius for different thicknesses of the biomolecular coating: (a) dipolar LSPR peak, (b) quadrupolar LSPR peak. 

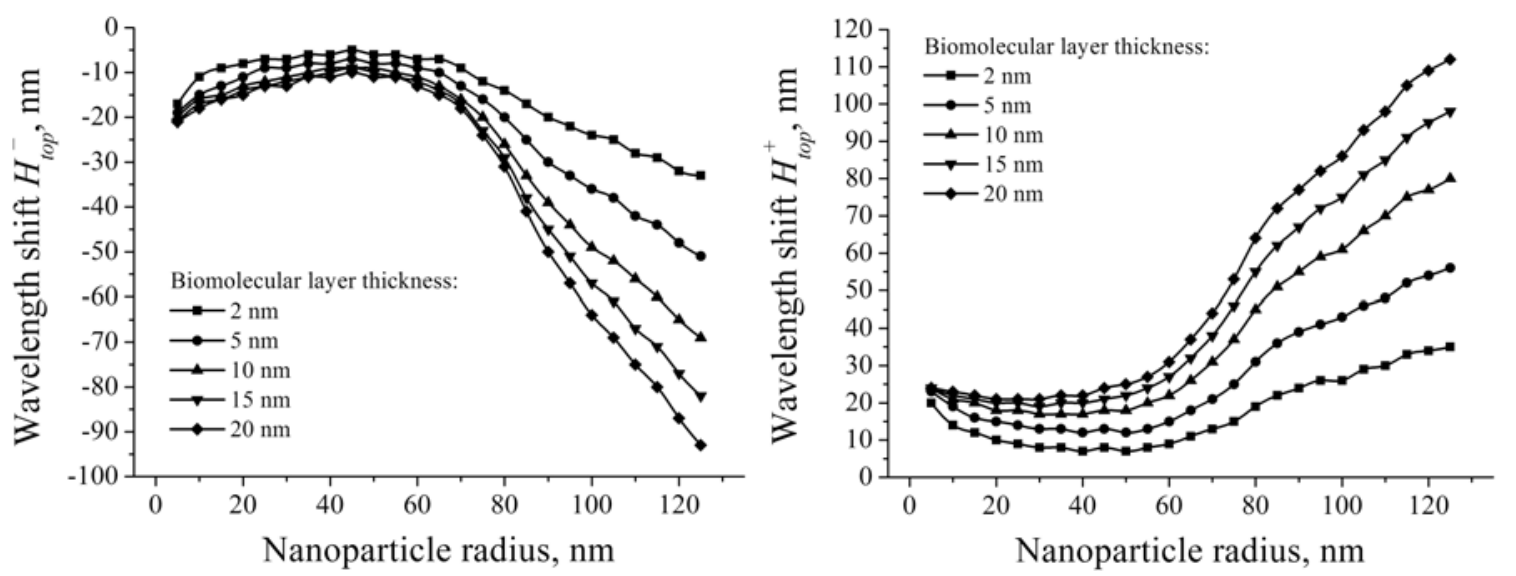

Fig. 5. LSPR responses (a) $H_{\text {top }}^{-}$and (b) $H_{\text {top }}^{+}$versus nanoparticle radius for different thicknesses of the biomolecular coating, measured on the dipolar LSPR peak.
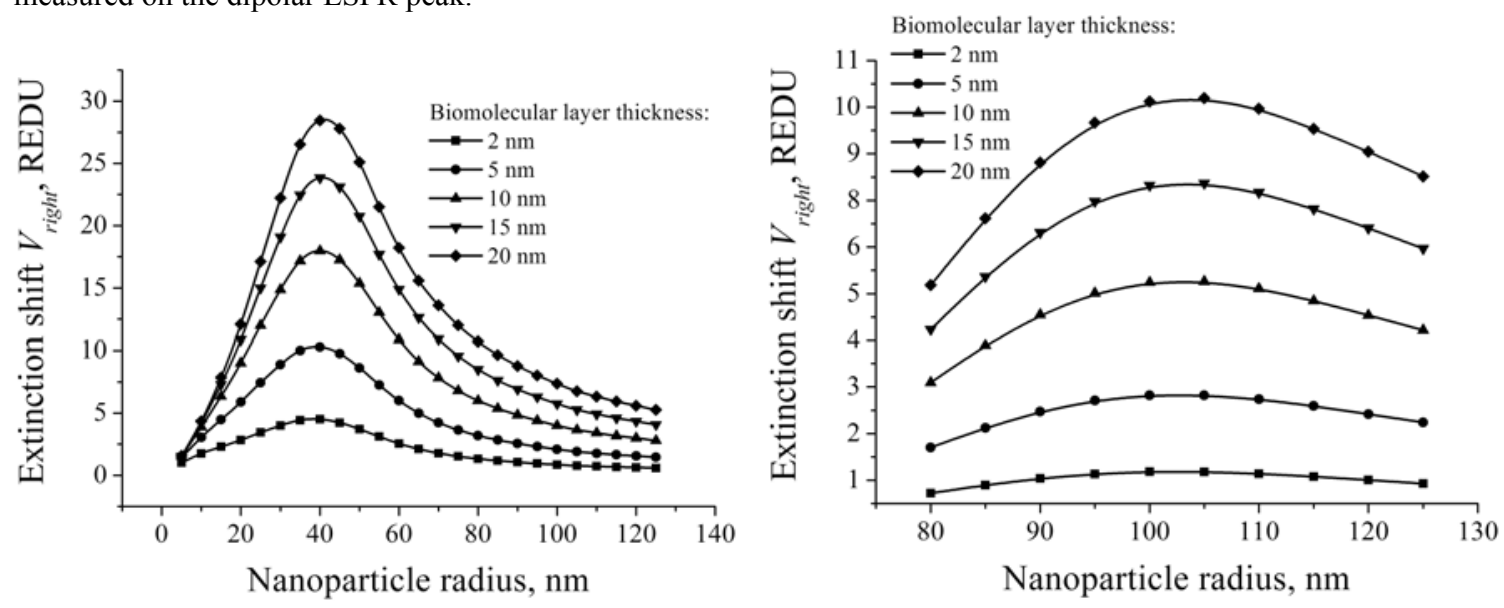

Fig. 6. LSPR response $V_{\text {right }}$ versus nanoparticle radius for different thicknesses of the biomolecular coating: (a) dipolar LSPR peak, (b) quadrupolar LSPR peak.

When studying the "vertical" LSPR responses, a problem with a measurement unit selection arises. Theoretical modeling yields dimensionless extinction efficiency, while experimental measurements produce signal in units of absorbance, which is dependent on the sample structure. This fact stimulated us to introduce new relative unit of LSPR response measurement, relative extinction difference unit (REDU), which will serve as a basis for the measurement of $V_{\text {left }}, V_{\text {top }}$, $V_{\text {right }}$ and $V_{\max }$ LSPR responses. We have established the $V_{\text {right }}$ response for a gold nanoparticle with a radius of $10 \mathrm{~nm}$ and $1 \mathrm{~nm}$ thick biomolecular shell, which is 0.04631 in units of extinction efficiency, as equal to 1 REDU. As a result of "vertical" LSPR responses comparison, we found out that the maximal response is provided by the $V_{\text {right }}$ measurement mode both for dipolar and quadrupolar peak for all biomolecule sizes studied. The dependences of $V_{\text {right }}$ on the nanoparticle size for different biomolecular cover thicknesses are presented in Fig. 6. Clearly, absolute maximal response for all biomolecule sizes discussed is provided by the $V_{\text {right }}$ measurement on the dipolar LSPR peak. At the same time, for the nanoparticle radius over $90 \mathrm{~nm} V_{\text {right }}$ response on the quadrupolar peak overtops the one on the dipolar peak. It should be noted that response curves for both dipolar and quadrupolar LSPR peaks exhibit maxima (see Fig. 6), which correspond to the optimal nanoparticle size for the detection of the biomolecular overlayer with a maximum response. Respective nanoparticle radii are about $40 \mathrm{~nm}$ for the dipolar LSPR peak and near 100-105 $\mathrm{nm}$ for the quadrupolar one.

Interestingly, how successful was our selection of the point on the right slope of the LSPR peak for the measurement of the $V_{\text {right }}$ response. It turned out that the point selected for $V_{\text {right }}$ measurement mode got near the point where the maximal extinction shift $V_{\max }$ is produced. This is evident from Fig. 7, where deviations of the LSPR response $V_{\text {right }}$ from $V_{\max }$ for dipolar and 

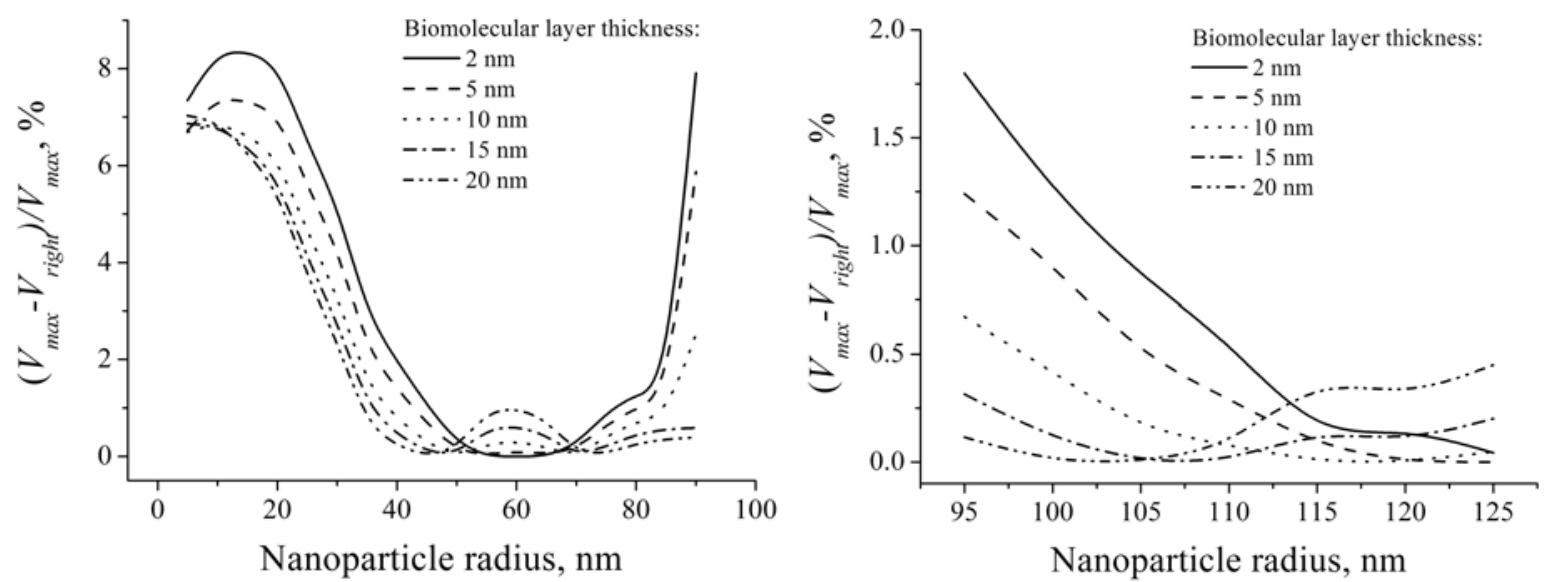

Fig. 7. Difference between the LSPR responses $V_{\text {right }}$ and $V_{\max }$ versus nanoparticle radius for different thicknesses of the biomolecular coating: (a) dipolar LSPR peak, (b) quadrupolar LSPR peak.

quadrupolar LSPR peaks are showed. The maximal difference between these responses is about $8 \%$ for dipolar peak and less than $2 \%$ for quadrupolar one, and corresponds to the case of a $2 \mathrm{~nm}$ thick biomolecular cover. This result points to a simple method for selection of an optimal point within the LSPR peak in the "vertical" response measurement mode - with acceptable accuracy, it can be chosen as a point of extreme spectrum derivative on a right slope of the peak.

\section{Conclusion}

We have theoretically studied a biosensor based on localized surface plasmon resonance in spherical $\mathrm{Au}$ nanoparticle by means of Mie theory for a spherical particle with a shell. Different response measurement modes that represent a formation of saturated biomolecular monolayer on the surface of the sensitive element have been considered in order to outline the optimal methods for LSPR response acquisition. Additionally, the sensitivity potential of dipolar and quadrupolar LSPR extinction peaks was compared, and optimal nanoparticle sizes for specific modes were designated.

New technique to measure "horizontal" LSPR response $H_{\text {top }}^{+}$was introduced and demonstrated to be more attractive for the application as LSPR biosensor response due to larger value of the wavelength shift provided in comparison with traditionally used extinction peak shift. Methods based on the measurement of wavelength shift turned out to give larger LSPR response when large-size Au nanoparticles are used as a sensitive element of biosensor for all biomolecule sizes investigated. Among "vertical" response measurement techniques, the optimal one was proved to be $V_{\text {right }}$, which is obtained at a wavelength of the extreme extinction spectrum derivative on a right slope of the LSPR peak. However, the size of the Au nanoparticle was demonstrated to influence the selection of the appropriate LSPR peak (dipole or quadrupole) in order to maximize the $V_{\text {right }}$ response. Optimal nanoparticle radii were found to be about $40 \mathrm{~nm}$ for the dipolar LSPR peak and near $100-105 \mathrm{~nm}$ for the quadrupolar one. Additionally, it was revealed that the introduced $V_{\text {right }}$ response is just few percent lower than maximum extinction difference $V_{\max }$ for studied coreshell configurations, thus implying the possible application of $V_{\text {right }}$ method as another promising one for LSPR biosensor response measurement.

\section{References}

1. C.R. Yonzon, E. Jeoung, S. Zou, G.C. Schatz, M. Mrksich, and R. P. Van Duyne, A comparative analysis of localized and propagating surface plasmon resonance sensors: the binding of concanavalin A to a monosaccharide functionalized self-assembled monolayer // J. Am. Chem. Soc. 126(39), p. 12669-12676 (2004).

2. A.J. Haes and R.P. Van Duyne, A unified view of propagating and localized surface plasmon resonance biosensors // Anal. Bioanal. Chem. 379(7-8), p. 920-930 (2004).

3. P. Englebienne, A. Van Hoonacker, M. Verhas, and N.G. Khlebtsov, Advances in high-throughput screening: biomolecular interaction monitoring in real-time with colloidal metal nanoparticles // Comb. Chem. High Throughput Screening 6(8), p. 777-787 (2003).

4. J.N. Anker, W.P. Hall, O. Lyandres, N.C. Shah, J. Zhao, and R.P. Van Duyne, Biosensing with plasmonic nanosensors // Nat. Mater. 7(6), p. 442453 (2008). 
5. E. Hutter and J.H. Fendler, Exploitation of localized surface plasmon resonance // Adv. Mater. 16(19), p. 1685-1706 (2004).

6. S. Lal, S. Link, and N.J. Halas, Nano-optics from sensing to waveguiding // Nat. Photonics 1(11), p. 641-648 (2007).

7. K. Aslan, J.R. Lakowicz, and C.D. Geddes, Plasmon light scattering in biology and medicine: new sensing approaches, visions and perspectives // Curr. Opin. Chem. Biol. 9(5), p. 538-544 (2005).

8. B. Sepúlveda, P.C. Angelomé, L.M. Lechuga, and L.M. Liz-Marzán, LSPR-based nanobiosensors // Nano Today 4(3), p. 244-251 (2009).

9. N. Nath and A. Chilkoti, A colorimetric gold nanoparticle sensor to interrogate biomolecular interactions in real time on a surface // Anal. Chem. 74(3), p. 504-509 (2002).

10. A.J. Haes and R.P. Van Duyne, A highly sensitive and selective surface-enhanced nanobiosensor, in Molecularly Imprinted Materials - Sensors and Other Devices, Mater. Res. Soc. Symp. Proc. 723, p. O3.1.1-O3.1.6, 2002.

11. N.G. Khlebtsov, Determination of size and concentration of gold nanoparticles from extinction spectra // Anal. Chem. 80(17), p. 6620-6625 (2008).

12. K.-S. Lee and M.A. El-Sayed, Gold and silver nanoparticles in sensing and imaging: sensitivity of plasmon response to size, shape, and metal composition // J. Phys. Chem. B 110(39), p. 1922019225 (2006).

13. A.J. Haes, S. Zou, G.C. Schatz, and R.P. Van Duyne, A nanoscale optical biosensor: the long range distance dependence of the localized surface plasmon resonance of noble metal nanoparticles // J. Phys. Chem. B 108(1), p. 109-116 (2004).

14. A.J. Haes, S. Zou, G.C. Schatz, and R.P. Van Duyne, Nanoscale optical biosensor: short range distance dependence of the localized surface plasmon resonance of noble metal nanoparticles // J. Phys. Chem. B 108(22), p. 6961-6968 (2004).

15. X. Liu, M. Atwater, J. Wang, and Q. Huo, Extinction coefficient of gold nanoparticles with different sizes and different capping ligands // Colloids Surf., B 58(1), p. 3-7 (2007).

16. M.D. Malinsky, K.L. Kelly, G.C. Schatz, and R.P. Van Duyne, Chain length dependence and sensing capabilities of the localized surface plasmon resonance of silver nanoparticles chemically modified with alkanethiol self-assembled monolayers // J. Am. Chem. Soc. 123(7), p. 1471$1482(2001)$.

17. J.J. Mock, D.R. Smith, and S. Schultz, Local refractive index dependence of plasmon resonance spectra from individual nanoparticles // Nano Lett. 3(4), p. 485-491 (2003).

18. W.A. Murray, B. Auguie, and W.L. Barnes, Sensitivity of localized surface plasmon resonances to bulk and local changes in the optical environment // J. Phys. Chem. C 113(13), p. 51205125 (2009).

19. M.M. Miller and A.A. Lazarides, Sensitivity of metal nanoparticle surface plasmon resonance to the dielectric environment // J. Phys. Chem. B 109(46), p. $21556-21565$ (2005).

20. W.P. Hall, J.N. Anker, Y. Lin, J. Modica, M. Mrksich, and R.P. Van Duyne, A CalciumModulated Plasmonic Switch // J. Am. Chem. Soc. 130(18), p. 5836-5837 (2008).

21. Y. Wang, J. Deng, J. Di, and Y. Tu, Electrodeposition of large size gold nanoparticles on indium tin oxide glass and application as refractive index sensor // Electrochem. Comm. 11(5), p. 1034-1037 (2009).

22. X. Li, L. Jiang, Q. Zhan, J. Qian, and S. He, Localized surface plasmon resonance (LSPR) of polyelectrolyte-functionalized gold-nanoparticles for bio-sensing // Colloids Surf., A 332(2-3), p. 172-179 (2009).

23. C.R. Yonzon, D.A. Stuart, X. Zhang, A.D. McFarland, C.L. Haynes, and R.P. Van Duyne, Towards advanced chemical and biological nanosensors - An overview // Talanta 67(3), p. 438-448 (2005).

24. Y.-Q. Chen and C.-J. Lu, Surface modification on silver nanoparticles for enhancing vapor selectivity of localized surface plasmon resonance sensors // Sens. Actuators, B 135(2), p. $492-498$ (2009).

25. C.-S. Cheng, Y.-Q. Chen, and C.-J. Lu, Organic vapour sensing using localized surface plasmon resonance spectrum of metallic nanoparticles self assemble monolayer // Talanta 73(2), p. 358-365 (2007)

26. V. Chegel, B. Lucas, J. Guo, A. Lopatynskyi, O. Lopatynska, and L. Poperenko, Detection of biomolecules using optoelectronic biosensor based on localized surface plasmon resonance. Nanoimprint lithography approach // Semicond. Phys. Quantum Electron. Optoelectron. 12(1), p. 91-97 (2009).

27. Y. Zhou, H. Xu, A.B. Dahlin, J. Vallkil, C.A.K. Borrebaeck, C. Wingren, B. Liedberg, and F. Höök, Quantitative interpretation of gold nanoparticlebased bioassays designed for detection of immunocomplex formation // Biointerphases 2(1), p. 6-15 (2007).

28. K.-J. Chen and C.-J. Lu, A vapor sensor array using multiple localized surface plasmon resonance bands in a single UV-vis spectrum // Talanta 81(45), p. 1670-1675 (2010).

29. D.W. Lynch and W.R. Hunter, Comments on the optical constants of metals and an introduction to the data for several metals, in Handbook of optical constant of solids, Vol. 1, Ed. E.D. Palik, p. 275 368, Academic Press, San Diego, CA, 1998.

30. P.B. Johnson and R.W. Christy, Optical constants of the noble metals // Phys. Rev. B 6(12), p. 43704379 (1972). 
31. L.I. Berger, Fermi energy and related properties of metals, in Handbook of chemistry and physics, 84th ed., Ed. R.D. Lide, p. 12-232-12-233, CRC Press, Boca Raton, FL, 2004.

32. A.I. Gusev and A.A. Rempel, Nanocrystalline Materials, Nauka, Moscow, Russian Federation, 2001 (in Russian).

33. U. Kreibig and M. Vollmer, Optical Properties of Metal Clusters (Springer Series in Materials Science, vol. 25), Springer, Berlin, Germany, 1995.

34. N.L. Dmitruk, A.V. Goncharenko, and E.F. Venger, Optics of Small Particles and Composite Media, Naukova dumka, Kyiv, Ukraine, 2009.

35. S.L. Westcott, J.B. Jackson, C. Radloff, and N.J. Halas, Relative contributions to the plasmon line shape of metal nanoshells // Phys. Rev. B 66(15), p. 155431-1-155431-5 (2002).

36. M. Fox, Optical properties of solids, Oxford University Press, New York, NY, 2001.

37. R.A. Paquin, Properties of metals, in Handbook of optics, Vol. 2 - Devices, Measurements, and Properties, 2nd ed., Ed. M. Bass, p. 35.1-35.78, McGraw-Hill, New York, NY, 1995.
38. H. Tokuhisa, M. Zhao, L.A. Baker, V.T. Phan, D.L. Dermody, M.E. Garcia, R.F. Peez, R.M. Crooks, and T.M. Mayer, Preparation and characterization of dendrimer monolayers and dendrimer-alkanethiol mixed monolayers adsorbed to gold // J. Am. Chem. Soc. 120(18), p. 4492-4501 (1998).

39. M. Weisser, G. Tovar, S. Mittler-Neher, W. Knoll, F. Brosinger, H. Freimuth, M. Lacher, and W. Ehrfeld, Specific bio-recognition reactions observed with an integrated Mach-Zehnder interferometer // Biosens. Bioelectron. 14(4), p. 405-411 (1999).

40. N.G. Khlebtsov, V.A. Bogatyrev, B.N. Khlebtsov, L.A. Dykman, and P. Englebienne, A multilayer model for gold nanoparticle bioconjugates: application to study of gelatin and human IgG adsorption using extinction and light scattering spectra and the dynamic light scattering method // Colloid J. 65(5), p. 622-635 (2003).

41. C.F. Bohren and D.R. Huffman, Absorption and scattering of light by small particles, WileyInterscience, New York, NY, 1983. 